\title{
Is It Possible to Train Surgeons for Rural Africa? Reply
}

\author{
Stephen Merry
}

Published online: 11 February 2012

(c) Société Internationale de Chirurgie 2012

I am grateful for Dr. Chu's leadership in the promotion of task-shifting to heal the "neglected tropical disease" of surgical illnesses in resource-limited settings. While her letter affirms the feasibility and necessity of training and employing general medical officers at district hospitals to provide primary surgical services, it also clarifies some of the challenges ahead.

Clearly, individual competency does not equate with institutional competency in providing essential surgical services. The real and perceived impediments are broad and deep and illuminate potential misunderstandings, inaction, or powerlessness at multiple levels. Relevant to our discussion of surgical task-shifting, while we may broaden individual surgical competencies of technicians and generalists, lower institutional competencies and structural failures in the health system may prevent progress. Neither a well-trained general surgeon nor a medical officer/family physician with additional surgical training will easily overcome a lack of essential medicines, anesthesia or surgical support staff, and sufficient qualified nurses for recovery care.

Although fully trained general surgeons may rightly consider themselves the "cornerstone to improving surgical care in rural Africa" [1,2], implicit in that analogy is the image of a building mostly composed of other stonestechnicians, nurses, and generalists-all sharing in the surgical load. What I was pleading for in my letter to the editor [3], and what the Primary Surgery texts of Maurice King et al., the WHO Global Initiative on Emergency and Essential Surgical Care (GIEESC) [4] and the fine GIEESC

S. Merry $(\square)$

Department of Family Medicine, Mayo Clinic,

Rochester, MN, USA

e-mail: merry.stephen@mayo.edu manual Surgical Care at the District Hospital e-learning toolkit and training videos, and the papers on surgical taskshifting by Dr. Chu and others referenced in my prior communication call us to is a recognition that neither general surgeons nor any other isolated group will alone solve the global scourge of surgical illness morbidity and mortality. Moreover, these resources call us to recognize essential surgery as part of primary health care, as a fundamental need, as a human right to be guaranteed by each country's public health policy. Simplified to its component parts, this goal for surgery is achievable in even the most resource-limited settings. The published resources cited above also remind us that, with care providers working together to form a team at the upper limits of our competencies, healing is within reach. I am grateful to my surgical colleague for affirming this hope.

\section{References}

1. Pollock JD, Love TP, Steffes BC et al (2011) Is it possible to train surgeons for rural Africa? A report of a successful international program. World J Surg 35:493-499. doi:10.1007/s00268-010-09 36-Z

2. Pollock JD, Love TP, Steffes BC et al (2011) Is it possible to train surgeons for rural Africa? A report of a successful international program: Reply. World J Surg 35:2175-2176. doi:10.1007/s00 268-011-1189-1

3. Merry SP (2011) Is it possible to train surgeons for rural Africa? A report of a successful international program: Letter to the Editor. World J Surg 35:2172-2174. doi:10.1007/s00268-011-1154-Z

4. WHO (2011) Global initiative for Emergency and Essential Surgical Care (GIEESC). http://www.who.int/surgery/globalinitiative/ en. Accessed 20 Dec 2011 